\title{
Voltage Stability Constrained Optimal Power Flow Using NSGA-II
}

\author{
Sandeep Panuganti ${ }^{1}$, Preetha Roselyn John ${ }^{1}$, Durairaj Devraj ${ }^{2}$, Subhransu Sekhar Dash ${ }^{1}$ \\ ${ }^{1}$ Department of Electrical and Electronics Engineering, Sri RamaswamyMemorial, Chennai, India \\ ${ }^{2}$ DEAN (Research), Kalasalingam University, Krishnankoil, India \\ Email: sandeep26psk@gmail.com, preetha.roselyn@gmail.com
}

Received November 30, 2012; revised December 30, 2012; accepted January 8, 2013

\begin{abstract}
Voltage stability has become an important issue in planning and operation of many power systems. This work includes multi-objective evolutionary algorithm techniques such as Genetic Algorithm (GA) and Non-dominated Sorting Genetic Algorithm II (NSGA-II) approach for solving Voltage Stability Constrained-Optimal Power Flow (VSC-OPF). Base case generator power output, voltage magnitude of generator buses are taken as the control variables and maximum L-index of load buses is used to specify the voltage stability level of the system. Multi-Objective OPF, formulated as a multi-objective mixed integer nonlinear optimization problem, minimizes fuel cost and minimizes emission of gases, as well as improvement of voltage profile in the system. NSGA-II based OPF - case 1-Two objective-Min Fuel cost and Voltage stability index; case 2-Three objective-Min Fuel cost, Min Emission cost and Voltage stability index. The above method is tested on standard IEEE 30-bus test system and simulation results are done for base case and the two severe contingency cases and also on loaded conditions.
\end{abstract}

Keywords: Voltage Stability; Optimal Power Flow; Multi Objective Evolutionary Algorithms

\section{Introduction}

GA, invented by Holland in the early 1970 s, is a stochastic global search method that mimics the metaphor of natural biological evaluation.Genetic Algorithms (GA) [1] operates on a population of candidate solutions encoded to finite bit string called chromosome. In order to obtain optimality, each chromosome exchanges the information using operators borrowed from natural genetic to produce the better solution. The combined Economic-Emission multiobjective problem seeks to simultaneously minimize both fuel costand the emissions produced by power plants. Environmental concerns on the effect of $\mathrm{SO}_{2}$ and NOX emissions producedby the fossil-fueled power plants led to the inclusion ofminimization of emissions as an objective in the OPF formulation.

\subsection{Voltage Stability}

Voltage instability stems from the attempt of load dynamics to restore power consumption beyond the capability of the combined transmission and generation. Voltage stability constrained OPF-Voltage stability indicator is incorporated in the OPF formulation through the L-index value. The voltage stability index is an appropriate measure of the closeness of the system to voltage collapse. NSGA-II is a popular non-domination based genetic algorithm for multi-objective optimization which has a better sorting algorithm and incorporates elitism and no sharing parameter needed to be chosen as compared to the original NSGA. Emission cost of generators also play a vital role and is thus formulated in the minimization OPF problem. Since OPF was introduced in 1968, several methods have been employed to solve this problem, e.g. Gradient base, Linear programming method and Quadratic programming. However all of these methods suffer from three main problems. Firstly, they may not be able to provide optimal solution and usually getting stuck at local optima [2]. Secondly, all these methods are based on assumption of continuity and differentiability of objective function which is not actually allowed in a practical system.

\subsection{VSC-OPF}

The Contingencies such as unexpected line outages in a stressed system may often result in voltage instability, which may lead to voltage collapse. After a voltage collapse, the system becomes dismantled owing to the widespread operation of protective devices. Studies have been performed to predict the voltage instability with both static and dynamic approaches.

In this paper three different cases along with the system loaded conditions are considered. In the first case 
base case OPF as a single objective optimization problem is solved using GA [3]. In the second case VSC-OPF problem is formulated in MOGA with minimization of fuel cost and L-index value. In the third case economic emission of gases along with VSC-OPF problem is considered as a multi-objective problem and L-index is solved using the NSGA-II approach in an IEEE 30 bus system. NSGA [4] is a popular non-domination based genetic algorithm for multi-objective optimization. It is a very effective algorithm but has been generally criticized for its computational complexity, lack of elitism and for choosing the optimal parameter value for sharing parameter $\sigma$ share. A modified version, NSGA-II [5] was developed, which has a better sorting algorithm, incurporates elitism and no sharing parameter needs to be chosen a priori.

\section{Voltage Stability Index}

The voltage stability analysis involves determination of an index known as voltage collapse proximity indicator. This index is an approximate measure of the closeness of the system to voltage collapse. There are various methods of determining the voltage collapse proximity indicator. One such method is the L-index method proposed in Kessel and Glavitsch. It is based on load flow analysis. Its value ranges from 0 (no load condition) to 1 (voltage collapse). The bus with the highest L-index value will be the most vulnerable bus in the system. The technique is incorporated from [6]. The L-index calculation for a power system is briefly discussed below.

Consider an N-bus system in which there are $N_{g}$ generators. The relationship between voltage and current can be expressed by the following expression:

$$
I_{\text {bus }}=Y_{\text {bus }} \cdot V_{\text {bus }}
$$

By segregating the load buses (PQ) from generator buses (PV), Equation (1) can write as

$$
\left[\begin{array}{c}
I_{G} \\
I_{L}
\end{array}\right]=\left[\begin{array}{ll}
Y_{G G} & Y_{G L} \\
Y_{L G} & Y_{L L}
\end{array}\right]\left[\begin{array}{c}
V_{G} \\
V_{L}
\end{array}\right]
$$

where $I_{G}, I_{L}$ and $V_{G}, V_{L}$ represent currents and voltages at the generator buses and load buses.

Rearranging the above equation we get:

$$
\left[\begin{array}{c}
V_{L} \\
I_{G}
\end{array}\right]=\left[\begin{array}{ll}
Z_{L L} & F_{L G} \\
K_{G L} & Y_{G G}
\end{array}\right]\left[\begin{array}{c}
I_{L} \\
V_{G}
\end{array}\right]
$$

where:

$$
F_{L G}=-\left[Y_{L L}\right]^{-1}\left[Y_{L G}\right]
$$

The L-index of the $j^{\text {th }}$ node is given by the expression:

$$
L_{j}=\left|1-\sum_{i=1}^{N_{g}} F_{J I} \frac{V_{i}}{V_{j}} \angle\left(\theta_{j i}+\delta_{i}-\delta_{j}\right)\right|
$$

where:

\begin{tabular}{cc}
\hline$V_{i}$ & Voltage magnitude of $i$ th generator \\
$V_{j}$ & Voltage magnitude of $j^{\text {th }}$ generator \\
$\theta_{\mathrm{ji}}$ & Phase angle of the term $F_{j i}$ \\
$\delta_{\mathrm{i}}$ & Voltage phase angle of $i^{\text {th }}$ generator unit \\
$\delta_{\mathrm{j}}$ & Voltage phase angle of $j^{\text {th }}$ generator unit \\
$N_{g}$ & Number of generating units. \\
\hline
\end{tabular}

$V_{L}, I_{L}$ : Voltages and Currents for PQ buses; $V_{G}, I_{G}$ : Voltages and Currents for PV buses; Where, $Z_{L L}, F_{L G}, K_{G L}, Y_{G G}$ : sub matrices generated from $Y_{b u s}$ partial inversion.

\section{$L_{j}$ : L-index voltage stability indicator for bus $k$.}

The values of $F_{j i}$ are obtained from the matrix $F_{L G}$. The L-indices for a given load condition are computed for all the load buses and the maximum of the L-indices $\left(L^{\max }\right)$ gives the proximity of the system to voltage collapse. The L-index has the advantage of indicating voltage instability proximity of the current operating point without calculation of the information about the maximum loading point.

\section{Problem Formulation}

In general, the OPF problem is formulated as an optimisation problem in which a specific objective function is minimised while satisfying a number of equality and inequality constraints[7]. The objectives of the OPF problem considered here are minimisation of fuel cost in the normal state and the minimisation of the voltage stability index $L^{\max }$ in the emergency state. Power flow equations are the equality constraints of the problem, while the inequality constraints include the limits on real and reactive power generation and bus voltage magnitude as follows.

$$
\begin{gathered}
\text { Minimise } F_{1}=\sum_{i=1}^{N_{G}}\left(a_{i} P_{G i}+b_{i} P_{G i}+c_{i}\right) \\
\text { Minimise } F_{2}=\sum L^{\text {max. }} \\
\text { Minimise } F_{3}=\sum_{i=1}^{N_{G}}\left(d_{i} P_{G i}+e_{i} P_{G i}+g_{i}\right) \\
\text { Inequality constraints } P_{G i}^{\text {min. }}{ }_{G i} \leq P_{G i} \leq P_{G i}^{\text {max. }} \\
V_{i}^{\text {min. }} \leq V_{i} \leq V_{i}^{\text {max. }} \\
Q_{G i}^{\text {min. }} \leq Q_{G i} \leq Q_{G i}^{\text {max. }}
\end{gathered}
$$

where:

$$
\text { Equality Constraints } P_{G}=P_{D}
$$

$N$ the number of total buses

$N_{G}$ the number of generator buses

$N_{L}$ the number of load buses

$N_{b}$ the number of transmission lines

$P_{i}, Q_{i}$ real and reactive power injected at bus $i$

$\left|V_{i}\right|$ Voltage magnitude at bus $i$ 
The equality constraints given by the above equations are satisfied by running the power flow program. The active power generation $\left(P_{g i}\right)$ (except the generator at the slack bus) and generator terminal bus voltages $\left(V_{g i}\right)$ are the optimization variables and they are self-restricted by the optimization algorithm.

\section{Non-Dominated Sorting Genetic Algorithm II (NSGA-II)}

NSGA introduced by Srinivas and Deb [8], implements the idea of a selection method based on classes of dominance of all solutions. This algorithm identifies nondominated solutions in the population, at each generation, to form non-dominated fronts, based on the concept of non-dominance of Pareto. After this, the usual selection, crossover, and mutation operators are performed.

However, there are some disadvantages in NSGA. It has been generally criticized for its computational complexity, lack of elitism and for choosing the optimal parameter value for sharing parameter $\sigma_{\text {share }}$. A modified version, NSGA-II was developed, which has a better sorting algorithm, incorporates elitism and no sharing parameter needs to be chosen a priori [9]. In this algorithm, the population is initialized as random, and the number of population is $\mathrm{N}$. Once the population in initialized the population is sorted based on non-domination into each front. The first front being completely non-dominant set in the current population and the second front being dominated by the individuals in the first front only and the front goes so on. Each Individual in the each front are assigned rank values or based on front in which they belong to. Then, crowding distance is calculated for each individual. The crowding distance is a measure of how close an individual is to its neighbours.

The NSGA-II procedure is also shown in Figure 1. Parents are selected from the population by using binary tournament selection based on the rank and crowding distance. The individual with lesser rank or greater crowding distance is selected. The selected population generates offspring from crossover and mutation operators. The population with the current population and current offspring is sorted again based on non-domination

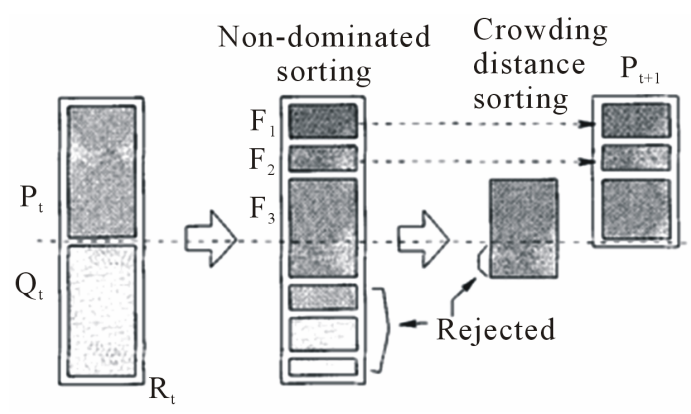

Figure 1. NSGA-II procedure. and only the best $N$ individuals are selected. The selection is based on rank and on crowding distance on the last front. Then the new population will be selected as parents at the next round.

\subsection{Population Initialization}

The population is initialized based on the problem range and constraints if any.

\subsection{Non-Dominated Sort}

The The initialized population is sorted based on nondomination. The fast sort algorithm is described as below.

- For each individual $p$ in main population $P$ do the following

Initialize $S p=\phi$. this set would contain all the individuals that are being dominated by $p$.

Initialize $n_{p}=0$. This would be the number of individuals that dominate $p$.

For each individual $q$ in $P$

If $p$ dominated $q$ then

Add $q$ to the set $S_{p}$

Else if $q$ dominated $\mathrm{p}$ then

Increment the dominated counter for $\mathrm{p}$ i.e. $n_{p}=n_{p}+1$. If $n_{p}=0$ i.e. no individual dominate $p$ then $p$ belongs to the first front, set rank of individual $p$ to one i.e. $p_{\text {rank }}=1$. Update the first front set by adding $\mathrm{p}$ to front one i.e. $F_{1}=F_{1} \cup\{p\}$

- This is carried out for all the individuals in main population $P$.

- Initialize the front counter to one $I=1$.

- Following is carried out while the $i^{\text {th }}$ front is nonempty i.e. $F i \neq \phi$.

$Q=\phi$. The set for storing the individuals for $(i+1)^{\text {th }}$ front.

For each individual $p$ in front $F_{i}$

For each individual $q$ in $S_{p}\left(S_{p}\right.$ is the set of individuals dominated by $p$ )

$n_{q}=n_{q}-1$, decrement the domination count for individual $q$.

If $n_{q}=0$ then none of the individuals in the subsequent fronts would dominate q. hence set $q_{\text {rank }}=i+1$. Update the set $Q$ with individual $q$ i.e. $Q=Q \cup q$.

Increment the front counter by one.

Now the set $Q$ is the next front and hence $F_{i}=Q$.

This algorithm is better than NSGA [10] since it utilize the information about the set that an individual dominate $\left(S_{p}\right)$ and number of individuals that dominate the individual $\left(n_{p}\right)$.

\subsection{Crowding Distance}

Once the non-dominated sort is complete the crowding distance is assigned. Since the individuals are selected based on rank and crowding distance all the individuals 
in the population are assigned a crowding distance value. Crowding distance is assigned front wise and comparing the crowding distance between two individuals in different front is meaningless. The crowing distance is calculated as below

- For each front $F i, n$ is the number of individuals.

- Initialize the distance to be zero for all the individuals i.e. $F i(d j)=0$, where $j$ corresponds to the $j^{\text {th }}$ individual in front $F i$.

- For each objective function $\mathrm{m}$.

- Sort the individuals in front $F i$ based on objective m i.e., $i=\operatorname{sort}(F i, m)$.

- Assign infinite distance to boundary values for each individual in $F_{i}$ i.e. $I\left(d_{1}\right)=\infty$ and $I(D n)=\infty$.

- For $k=2$ to $(n-1)$

$$
I\left(d_{k}\right)=I\left(d_{k}\right)+\frac{I(k+1) \cdot m-I(k-1) \cdot m}{f_{m}^{\max }-f_{m}^{\min }} .
$$

- $I(k) \cdot \mathrm{m}$ is the value of the $m^{\text {th }}$ objective function of the $k^{\text {th }}$ individual in $I$.

The basic idea behind the crowing distance is finding the euclidian distance between each individual in a front based on their $\mathrm{m}$ objectives in the $\mathrm{m}$ dimensional hyper space. The individuals in the boundary are always selected since they have infinite distance assignment.

\subsection{Selection}

Once the individuals are sorted based on non-domination and with crowding distance assigned, the selection is carried out using a crowded-comparison-operator $\left(a_{n}\right)$. The comparison is carried out as below based on

1) Non-domination rank $p_{\text {rank }}$ i.e. individuals in front $F_{i}$ will have their rank as $p_{\text {rank }}=i$.

2) Crowding distance $F_{i}\left(d_{j}\right)$

- $p a_{n} q$ if

- $p_{\text {rank }}<q_{\text {rank }}$

- or if $p$ and $\mathrm{q}$ belong to the same front $F_{i}$ then $F_{i}\left(d_{p}\right)>$ $F_{i}\left(d_{q}\right)$ i.e. the crowing distance should be more.

The individuals are selected by using a binarytournament selection with crowed-comparison-operator.

\subsection{Genetic Operators}

NSGA-II use Simulated Binary Crossover (SBX) $[10,11]$ and polynomial mutation $[10,12]$.

\subsubsection{Simulated Binary Crossover}

The Simulated binary crossover simulates the binary crossover observed in nature and is give as below.

$$
\left.\begin{array}{l}
C_{1, k}=\frac{1}{2}\left[\left(1-\beta_{k}\right) p_{1, k}+\left(1+\beta_{k}\right) p_{2, k}\right] \\
C_{2, k}=\frac{1}{2}\left[\left(1+\beta_{k}\right) p_{1, k}+\left(1-\beta_{k}\right) p_{2, k}\right]
\end{array}\right\}
$$

where $C_{i, k}$ is the $i^{\text {th }}$ child with $k^{\text {th }}$ component, $P_{i, k}$ is theselected parent and $\beta_{k}(\geq)$ is a sample from a random number generated having the density

$$
\left.\begin{array}{l}
p(\beta)=\frac{1}{2}\left(\eta_{c}+1\right) \beta^{\eta_{c}}, \text { if } 0 \leq \beta \leq 1 \\
p(\beta)=\frac{1}{2}\left(\eta_{c}+1\right) \frac{1}{\beta^{\eta_{c}+2}}, \text { if } \beta>1
\end{array}\right\}
$$

This distribution can be obtained from a uniformly sampled random number $u$ between $(0,1) . \eta_{\mathrm{c}}$ is the distribution index for crossover. That is

$$
\left.\begin{array}{l}
\beta(u)=(2 u)^{\frac{1}{(\eta+1)}} \\
\beta(u)=1 /[2(1-u)]^{\frac{1}{(\eta+1)}}
\end{array}\right\}
$$

\subsubsection{Polynomial Mutation}

$$
c_{k}=p_{k}+\left(p_{k}^{u}-p_{k}^{l}\right) \delta_{k}
$$

where $c_{\mathrm{k}}$ is the child and $p_{k}$ is the parent with $p_{k}^{u}$ being the upper boundon the parent component, $p_{k}^{l}$ is the lower bound and $\delta_{k}$ is small variation which is calculated from a polynomial distribution by using

$$
\left.\begin{array}{l}
\delta_{k}=\left(2 r_{k}\right)^{\frac{1}{\eta_{m}+1}}-1, \text { if } r_{k}<0.5 \\
\delta_{k}=1-\left[2\left(1-r_{k}\right)\right]^{\frac{1}{\eta_{m}+1}}, \text { if } r_{k} \geq 0.5
\end{array}\right\}
$$

$r_{k}$ is an uniformly sampled random number between $(0,1)$ and $\eta_{m}$ is mutation distribution index.

\subsection{Recombination and Selection}

The offspring population is combined with the current generation population and selection is performed to set the individuals of the next generation. Since all the previous and current best individuals are added in the population, elitism is ensured. Population is now sorted based on non-domination. The new generation is filled by each front subsequently until the population size exceeds the current population size. If by adding all the individuals in front $F j$ the population exceeds $N$ then individuals in front $F j$ are selected based on their crowding distance in the descending order until the population size is $N$. And hence the process repeats to generate the subsequent generations.

\section{Best compromised Solution}

Upon having the pareto-optimal set of non-dominated solution, the proposed approach [8] presents a best compromise solution tothe decision maker. Due to the imprecise nature of the decision maker's judgement, the $i^{\text {th }}$ objective function $J i$ is represented bya membership function defined as 


$$
\mu\left(J_{i}^{-}\right)=\left\{\begin{array}{cc}
1, & J_{i} \leq J_{i}^{\min } \\
\frac{J_{i}^{\max }-J_{i}^{-}}{J_{i}^{\max }-J_{i}^{\min }}, & J_{i}^{\min }<J_{i}^{-}<J_{i}^{\max } \\
0, & J_{i} \leq J_{i}^{\max }
\end{array}\right.
$$

where $J_{i}^{\max }$ and $J_{i}^{\min }$ are the maximum and minimum values of the $i^{\text {th }}$ objective function among all non-dominated solutions.

For each non-dominated solution $k$, the normalized membership function $\mu_{D}^{K}$ is calculated as

$$
\mu_{D}^{K}=\frac{\sum_{I=1}^{K} \mu\left(J_{i}^{K}\right)}{\sum_{k=1}^{K} \sum_{i=1}^{5} \mu\left(J_{i}^{K}\right)}
$$

\section{Simulation Results}

The proposed NSGA-II approach has been applied to solve the VSC-OPF problem in an IEEE 30-bus test system. The system has six generator buses, 24 load buses and 41 transmission lines. The generator cost coefficients and the transmission line parameters are taken from [12]. Three different cases were considered for simulation, one without considering the voltage stability i.e, to solve the VSC-OPF problem using MOGA and the second one is solved having economic emission of gases including VSC-OPF in NSGA-II.These simulations were implemented using the MATLAB program. The results of these simulations are presented, Figure 2.

In this case the two objectives are minimization of fuel cost and minimization of L-index using multi-objective Genetic Algorithm. The results of VSC-OPF using MOGA is shown in Table 1.

\section{1. (Case 1): VSC-OPF Using NSGA-II}

The voltage stability index (L-index) was included as the second objective function of the OPF problem along with the base fuel cost. The NSGA-II based algorithm was applied to solve this VSC-OPF problem. The optimal control variable setting obtained in this case is presented in Table 2 alongwith the L-index value. In Figure 4 shows the pareto optimal front of generation cost and L-index is shown and the Table 2 shows the line outage 27 - 28 along with line outage $27-30$ is shown in Table 3. The solution is a set of non-dominated solutions. The comparison of the results obtained in NSGA-II and three objective is shown in Table 5. From this table it is clear that the performance of NSGA-II is better than MOGA in VSC-OPF problem.

Contingency analysis was conducted on the system with $125 \%$ loaded condition by simulating the single line outages and in each case the maximum L-index value was evaluated. From the contingency analysis it was found that line outage $28-27$ is the most severe one
Table 1. NSGA-II base case.

\begin{tabular}{cccc}
\hline $\begin{array}{c}\text { Control } \\
\text { Variables }\end{array}$ & $\begin{array}{c}\text { Min F1 } \\
\text { (Fuel cost) }\end{array}$ & $\begin{array}{c}\text { Min F2 } \\
\text { (L-index) }\end{array}$ & $\begin{array}{c}\text { Best } \\
\text { compromised sol. }\end{array}$ \\
\hline P1 & 169.6545 & 140.9243 & 168.6642 \\
P2 & 50.0000 & 80.0000 & 50.4760 \\
P5 & 23.9893 & 42.9811 & 24.1108 \\
P8 & 22.0474 & 18.9395 & 22.1680 \\
P11 & 13.2559 & 17.0000 & 13.4598 \\
P13 & 14.8000 & 20.4000 & 14.8000 \\
V1 & 1.0000 & 1.0000 & 1.0000 \\
V2 & 1.0000 & 1.0040 & 1.0000 \\
V5 & 0.9826 & 1.0000 & 0.9991 \\
V8 & 0.9884 & 1.0000 & 0.9891 \\
V11 & 0.9899 & 0.9903 & 0.9918 \\
V13 & 0.9874 & 0.9888 & 0.9933 \\
Fuel cost, F1 & 807.1765 & 872.7911 & 807.9227 \\
L-index , F2 & 0.1101 & 0.1075 & 0.1095 \\
\hline & & & \\
\hline
\end{tabular}

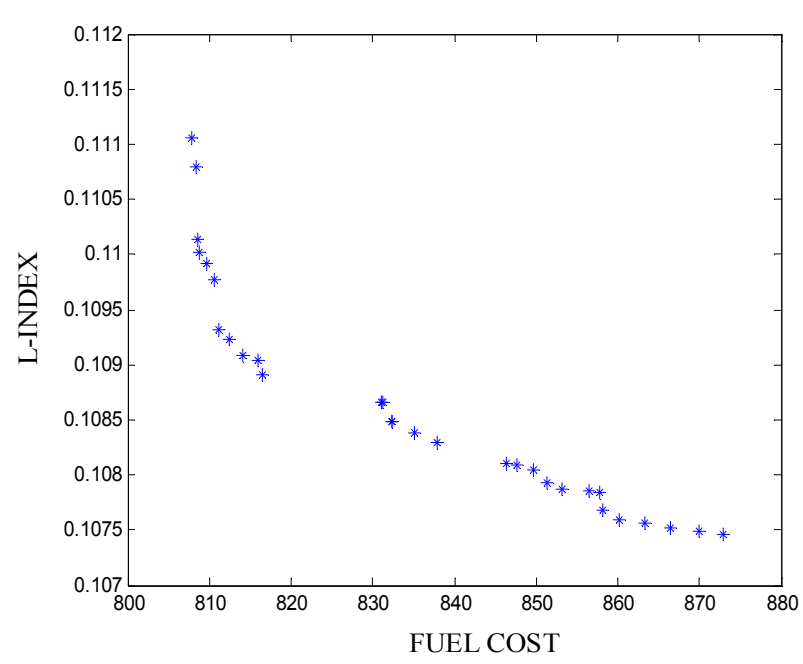

Figure 2. NSGA-II base case.

from the voltage security point of view during this contingency state.

Table 6 gives the fuel cost, $L^{\max }$ and minimum voltage value of the contingency constrained VSC-OPF using NSGA-II. This reduction in $L^{\max }$ is obtained at the expense of increased fuel cost. Figure 5 shows the pareto optimal front of contingency constrained VSC-OPF.

The line outage for 27 - 30 as shown in Figure 4, in Tables 4, 5 and is also performed along with the same loaded condition as in line outage $27-28$ as shown in Figure 3 and results are tabulated. The reduction in L-max is obtained at the extent of increased fuel cost. 
Table 2. NSGA II-Line outage 27 - 28.

\begin{tabular}{cccc}
\hline $\begin{array}{c}\text { Control } \\
\text { Variables }\end{array}$ & $\begin{array}{c}\text { Min F1 } \\
\text { (Fuel cost) }\end{array}$ & $\begin{array}{c}\text { Min F2 } \\
\text { (L-index) }\end{array}$ & $\begin{array}{c}\text { Best } \\
\text { compromised sol. }\end{array}$ \\
\hline P1 & 174.9058 & 112.3927 & 149.0853 \\
P2 & 50.000 & 80.0000 & 61.6056 \\
P5 & 22.0000 & 41.7412 & 27.7161 \\
P8 & 22.500 & 22.4915 & 19.6752 \\
P11 & 12.8957 & 16.1521 & 16.8888 \\
P13 & 14.8000 & 20.3995 & 20.4000 \\
V1 & 1.000 & 1.0000 & 1.0000 \\
V2 & 1.000 & 0.9901 & 0.9988 \\
V5 & 0.9874 & 0.9874 & 0.9856 \\
V8 & 0.9917 & 1.0040 & 0.9911 \\
V11 & 0.9903 & 0.9902 & 1.0000 \\
V13 & 0.9866 & 0.9866 & 0.9859 \\
Fuel cost, F1 & 814.0790 & 876.6776 & 814.2402 \\
L-index, F2 & 0.2905 & 0.2877 & 0.2895 \\
\hline
\end{tabular}

Table 3. NSGA-2-Line outage 27 - 30.

\begin{tabular}{cccc}
\hline $\begin{array}{c}\text { Control } \\
\text { Variables }\end{array}$ & $\begin{array}{c}\text { Min F1 } \\
\text { (Fuel cost) }\end{array}$ & $\begin{array}{c}\text { Min F2 } \\
\text { (L-index) }\end{array}$ & $\begin{array}{c}\text { Best } \\
\text { compromised sol. }\end{array}$ \\
\hline P1 & 173.2358 & 107.6728 & 147.3212 \\
P2 & 50.0000 & 80.0000 & 55.1566 \\
P5 & 22.2229 & 42.9728 & 30.5305 \\
P8 & 19.2142 & 22.5000 & 22.3421 \\
P11 & 15.1615 & 17.0000 & 17.0000 \\
P13 & 14.8000 & 20.4000 & 20.2354 \\
V1 & 1.0000 & 1.0000 & 1.0000 \\
V2 & 1.0000 & 0.9990 & 0.9984 \\
V5 & 0.9793 & 0.9876 & 0.9844 \\
V8 & 0.9866 & 1.0020 & 1.0020 \\
V11 & 0.9875 & 0.9930 & 0.9914 \\
V13 & 0.9835 & 0.9902 & 0.9886 \\
Fuel cost, F1 & 810.0262 & 876.3660 & 812.5044 \\
L-index, F2 & 0.1989 & 0.1953 & 0.1964 \\
\hline
\end{tabular}

\section{2. (Case 2): Economic Emission Based VSC-OPF Using NSGA-II}

The economic emission of the gases are included as the third objective along with the voltage stability index and

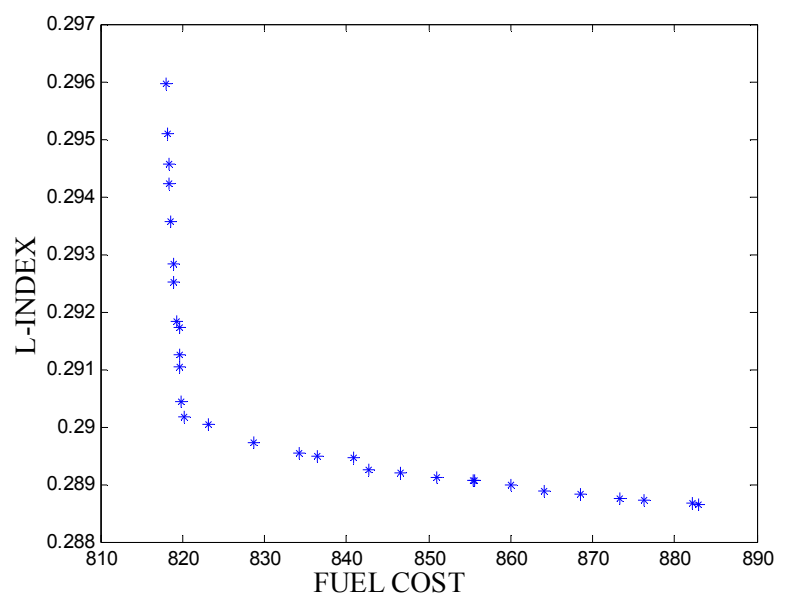

Figure 3. NSGA-II-Line outage 27 - 28.

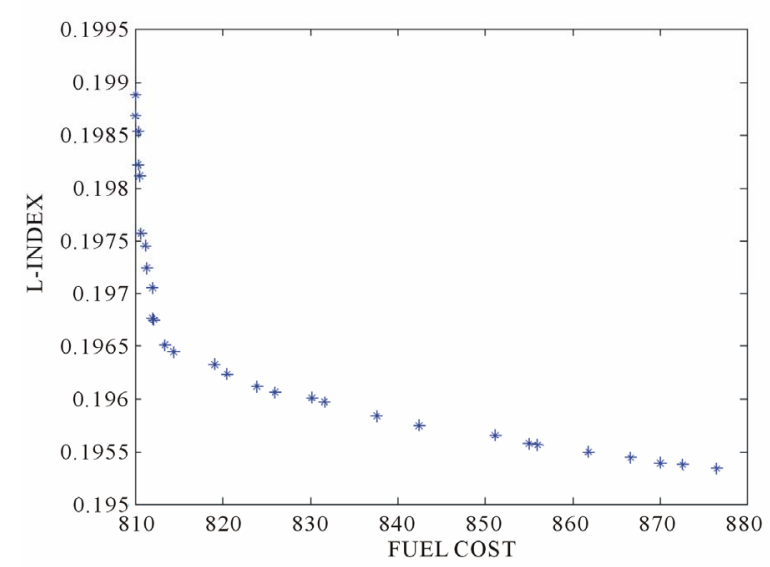

Figure 4. NSGA-2-Line outage 27 - 30.

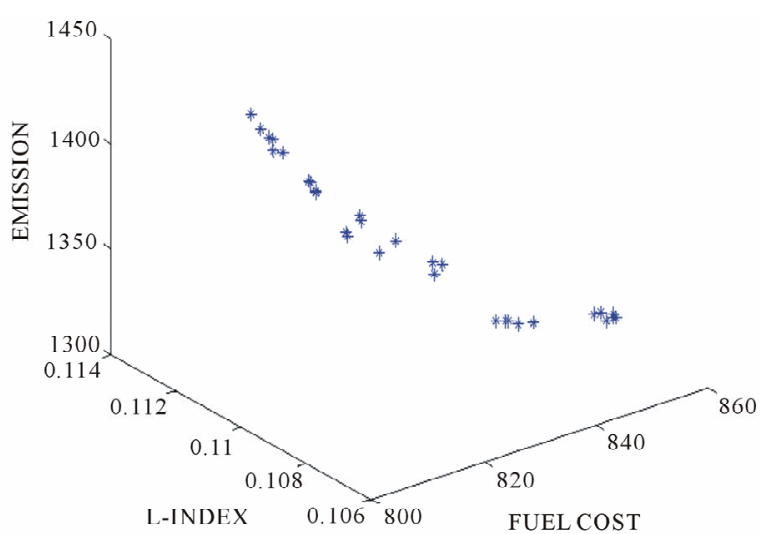

Figure 5. NSGA 2-3 Objective base case.

base fuel cost. The NSGA-II based algorithm was applied to solve this VSC-OPF problem. The optimal control variable settings are similar to that of the two objective case. In Figure 5 shows the pareto optimal front of generation cost, L-index and economic emission dispatch of gases for base case and in Figures 6, 7 the line outage 27 -28 and line outage $27-30$ are also included. 
Table 4. NSGA-II-3 objective line outage 27 - 28.

\begin{tabular}{|c|c|c|c|c|}
\hline $\begin{array}{l}\text { Control } \\
\text { Variables }\end{array}$ & $\begin{array}{c}\text { Min F1 } \\
\text { (Fuel cost) }\end{array}$ & $\begin{array}{c}\text { Min F2 } \\
\text { (L-index) }\end{array}$ & $\begin{array}{c}\text { Min } \\
\text { Emission, F3 }\end{array}$ & $\begin{array}{c}\text { Best } \\
\text { compromised sol. }\end{array}$ \\
\hline P1 & 160.3011 & 165.1097 & 169.5373 & 162.3011 \\
\hline P2 & 65.7505 & 60.3991 & 57.8956 & 66.7505 \\
\hline P5 & 23.3074 & 25.8083 & 22.000 & 24.3172 \\
\hline P8 & 16.6748 & 14.5257 & 15.4874 & 16.6738 \\
\hline P11 & 12.8745 & 13.0869 & 14.3629 & 12.6730 \\
\hline P13 & 14.8000 & 14.9005 & 14.8981 & 14.8230 \\
\hline V1 & 1.000 & 1.000 & 1.000 & 1.000 \\
\hline V2 & 1.000 & 1.000 & 1.000 & 1.000 \\
\hline V5 & 0.9999 & 1.000 & 0.9999 & 0.9999 \\
\hline V8 & 0.9999 & 1.000 & 0.9999 & 0.9899 \\
\hline V11 & 0.9999 & 1.000 & 1.000 & 0.9994 \\
\hline V13 & 0.9999 & 0.9999 & 1.000 & 0.9999 \\
\hline $\begin{array}{l}\text { Fuel cost, } \\
\text { F1 }\end{array}$ & 807.0019 & 853.6211 & 856.5133 & 809.0019 \\
\hline $\begin{array}{l}\text { L-index, } \\
\text { F2 }\end{array}$ & 0.1102 & 0.1077 & 0.1083 & 0.1099 \\
\hline $\begin{array}{l}\text { Emission, } \\
\text { F3 }\end{array}$ & 1424.5 & 1324.0 & 1316.2 & 1386.5 \\
\hline
\end{tabular}

Table 5. NSGA-2 - Line outage 27 - 30.

\begin{tabular}{|c|c|c|c|c|}
\hline $\begin{array}{l}\text { Control } \\
\text { Variables }\end{array}$ & $\begin{array}{c}\text { Min F1 } \\
\text { (Fuel cost) }\end{array}$ & $\begin{array}{c}\text { Min F2 } \\
\text { (L-index) }\end{array}$ & $\begin{array}{c}\text { Min } \\
\text { Emission, F3 }\end{array}$ & $\begin{array}{c}\text { Best } \\
\text { compromised sol. }\end{array}$ \\
\hline P1 & 170.8424 & 167.0531 & 145.6723 & 168.5707 \\
\hline P2 & 50.000 & 57.9636 & 69.0360 & 57.4857 \\
\hline P5 & 23.1042 & 23.8583 & 23.2324 & 23.0908 \\
\hline P8 & 21.3731 & 16.0485 & 20.4102 & 15.9655 \\
\hline P11 & 14.2896 & 12.6677 & 15.5218 & 12.8070 \\
\hline P13 & 14.8000 & 16.8955 & 19.3528 & 16.6933 \\
\hline V1 & 1.000 & 1.000 & 1.000 & 1.000 \\
\hline V2 & 1.000 & 1.000 & 1.000 & 1.000 \\
\hline V5 & 0.9989 & 1.000 & 0.9999 & 0.9999 \\
\hline V8 & 0.9883 & 0.9991 & 0.9999 & 1.000 \\
\hline V11 & 0.9914 & 1.000 & 0.9999 & 0.9999 \\
\hline V13 & 0.9925 & 0.9999 & 1.000 & 0.9999 \\
\hline $\begin{array}{l}\text { Fuel cost, } \\
\text { F1 }\end{array}$ & 809.9571 & 874.2141 & 844.7509 & 810.5175 \\
\hline $\begin{array}{l}\text { L-index } \\
\text { F2 }\end{array}$ & 0.1978 & 0.1944 & 0.1961 & 0.1954 \\
\hline $\begin{array}{c}\text { Emission, } \\
\text { F3 }\end{array}$ & 1413.7 & 1305.8 & 1325.7 & 1316.8 \\
\hline
\end{tabular}

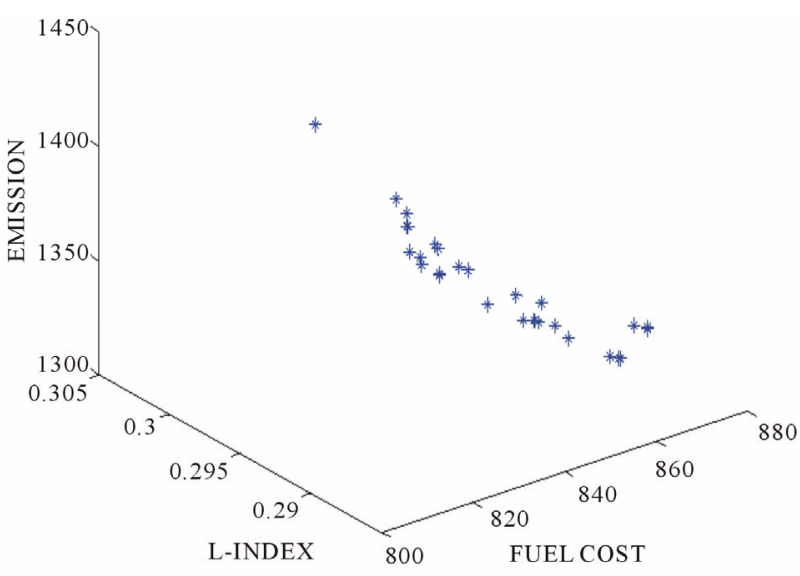

Figure 6. NSGA-2—Line outage 27 - 28.

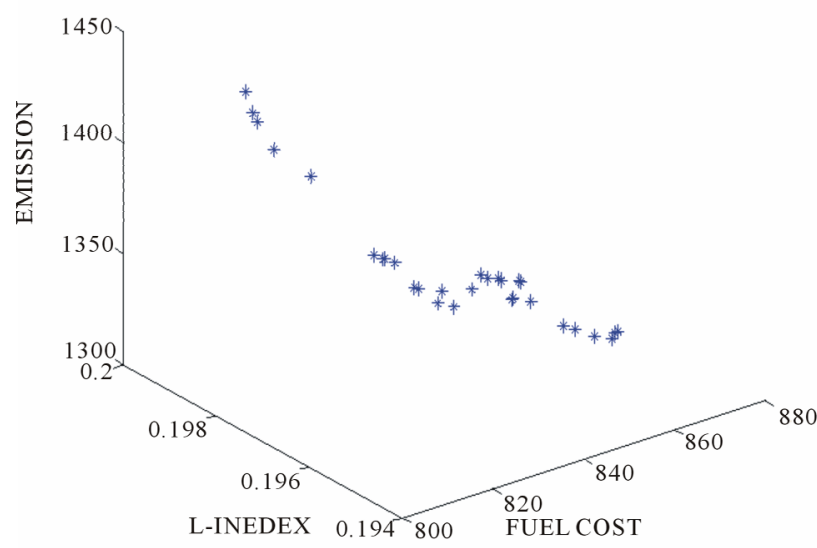

Figure 7. NSGA-2-Line outage 27 - 30.

\section{Conclusion}

In this paper, the various aspects of single-objective optimal power flow and multi-objective voltage stability constrained optimal power flow are studied. An efficient and diversified approach using NSGA-II algorithm is identified to solve the above multi-objective optimization problems. Several case studies have been employed separately for single \& multi-objective optimization problem. Firstly, the results are obtained for single objective OPF and contingency constrained VSC-OPF using genetic algorithm for the optimization of Fuel cost which are then compared with the power flow results of other papers. The multi-objective VSC-OPF problem is formulated using NSGA-II algorithm. The proposed algorithm occupies less memory space and takes CPU time than conventional GA approach. Simulation results of the IEEE 30-bus system have been presented to illustrate the effectiveness of the proposed approach to solve the VSC-OPF problem. This simulation results were carried out using NSGA-II and are found that voltage stability is 
improved in NSGA-II than multi-objective GA of the proposed algorithm than the other approaches.

\section{REFERENCES}

[1] N. Srinivas and K. Deb, "Multi-Objective Optimization Using Nondominated Sorting Ingenetic Algorithms," Technical Report, Department of Mechanical Engineering, Indian Institute of Technology, Kanpur, 1993.

[2] N. Srinivas and K. Deb, "Multi-Objective Optimization Using Nondominated Sortingin Genetic Algorithms," Evolutionary Computation, Vol. 2, No. 3, 1994, pp. 221-248. doi:10.1162/evco.1994.2.3.221

[3] A. J. Wood and B. F. Wollenberg, "Power Generation Operation and Control," John Wiley \& Sons, Inc., New York, 1996.

[4] M. S. Kumari, "Enhanced Genetic Algorithm Based Computation Technique for Multi-Objective, Optimal Power Flow Solution," Electrical Power and Energy Systems, Vol. 32, No. 6, 2010, pp. 736-742.

[5] K. O. Alawode, A. M. Jubril and O. A. Komolafe, "MultiObjective Optimal Power Flow Using Hybrid Evolutionary Algorithm," International Journal of Electrical Power \& Energy Systems Engin, Vol. 3, No. 3, 2010, p. 196.

[6] D. Devaraj and J. P. Roselyn, "Improved Genetic Algorithm for Voltage Security Constrained Optimal Power Flow Problem," International Journal of Energy Tech- nology and Policy, Vol. 5, No. 4, 2007, pp. 475-488. doi:10.1504/IJETP.2007.014888

[7] H. Sadat, "Power Systems Analysis," McGraw Hill Publication, New Delhi, 1997.

[8] O. Alsac, and B. Scott, "Optimal Load Flow with Steady State Security," IEEE Transactions on Power Systems, Vol. PAS-93, No. 3, 1974, pp.745-751. doi:10.1109/TPAS.1974.293972

[9] H. W. Dommel and W. F. Tinney, "Optimal Power Flow Solutions," IEEE Transactions on Power Apparatusand Systems, Vol. PAS-87, No. 10, 1968, pp. 1866-1876.

[10] S. Dhanalakshmi, S. Kannan, K. Mahadevan and S. Baskar, "Application of Modified NSGA-II Algorithm to Combined Economicand Emission Dispatch Problem," International Journal of Electrical Power \& Energy Systems, Vol. 33, No. 4, 2011, pp. 992-1002.

[11] R. He, G. A. Taylor and Y. H. Song, "Multi-Objective Optimal Reactive Power Flow including Voltage Security and Demand Profile Classification," International Journal of Electrical Power \& Energy Systems, Vol. 30, No. 5, 2008, pp. 327-336.

[12] K. O. Alawode, A. M. Jubril and O. A. Komolafe, "MultiObjective Optimal Reactive Power Flow Using Elitist Nondominated Sorting Genetic Algorithm: Comparison and Improvement," Journal of Electrical Engineering \& Technology, Vol. 5, No. 1, 2010, pp. 70-78. 